\title{
Development of Laser-driven Proton Source Toward Its Applications
}

\author{
Akito Sagisaka ${ }^{1 *}$, Hiroyuki Daido', Alexander S. Pirozhkov', Akifumi Yogo', \\ Koichi Ogura', Satoshi Orimo', Jinglong Ma', Michiaki Mori", Mamiko Nishiuchi", \\ Sergei V. Bulanov ${ }^{1,2,3}$, Timur Zh. Esirkepov ${ }^{1,3}$, Yuji Oishi ${ }^{4}$, Takuya Nayuki ${ }^{4}$, \\ Takashi Fujii ${ }^{4}$, Koshichi Nemoto ${ }^{4}$, and Hideo Nagatomo ${ }^{5}$ \\ ${ }^{1}$ Advanced Photon Research Center and Photo-Medical Research Center, Japan Atomic \\ Energy Agency, 8-1 Umemidai, Kizugawa-city, Kyoto 619-0215, Japan \\ ${ }^{2}$ A. M. Prokhorov Institute of General Physics of the Russian Academy of Sciences, \\ 38 Vavilov Street, 119991 Moscow, Russia \\ ${ }^{3}$ Moscow Institute of Physics and Technology, 9 Institutskiy pereulok, 141700 Dolgoprudny, \\ Moscow Region, Russia \\ ${ }^{4}$ Central Research Institute of Electronic Power Industry, 2-6-1 Nagasaka, \\ Yokosuka, Kanagawa 240-0196, Japan \\ ${ }^{5}$ Institute of Laser Engineering, Osaka University, 2-6 Yamadaoka Suita, \\ Osaka 565-0871, Japan
}

(Received January 2, 2009 : revised February 14, 2009 : accepted February 16, 2009)

We observe the proton signals produced by laser interaction with thin-foil targets of polyimide and of copper. We change the thickness of the polyimide target to $7.5 \mu \mathrm{m}, 12.5 \mu \mathrm{m}$, and $50 \mu \mathrm{m}$. High-energy protons with the maximum energy of $\sim 2.3 \mathrm{MeV}$ from $7.5 \mu \mathrm{m}$ thick polyimide are observed. This proton beam with the maximum energy of multi-MeV has various applications such as a proton shadowgraphy.

Keywords : High-intensity laser, Thin-foil target, Preformed plasma, ion, Proton acceleration

OCIS codes : (140.3460) Lasers; (120.3180) Interferometry; (350.5400) Plasmas; (350.4990) Particles

\section{INTRODUCTION}

High-energy ions produced in laser and thin foil interactions have been reported in a number of papers (see review [1] and references therein). A proton beam driven by a high-intensity laser is considered as a compact ion source for medical applications $[2,3]$. In order to develop the laser-driven proton beam, characterization of the proton energy distribution and beam divergence are important along with achievement of high energy. The energy spectra of those protons exhibit wide energy spread. The proton beams have large angular distribution of the order of $\sim 10^{\circ}$ with the source size of $\sim 10 \mu \mathrm{m}$. The laser-driven proton beams have extremely low transverse emittance [4-7], compared with those of the beams from the conventional accelerators.

*Corresponding author: sagisaka.akito@jaea.go.jp
These laser-driven beams can be applicable to a proton radiography system having high temporal and spatial resolutions [8], a source for the ion implantation [9] and a source for production of short-lived radioisotopes for medical and industrial applications $[10,11]$.

The preformed plasma plays an important role in the absorption of the main pulse. Kaluza et al. have reported that the optimal parameters of proton acceleration depend on the preformed plasma condition [12]. The experimental result indicated that the characterization of the preformed plasma is of essential importance for interpretation of ultra-short high-intensity laser-plasma interactions. Mackinnon et al. have reported that the maximum proton energy increases when the target thickness is reduced [13]. Neely et al. have observed high energy protons with thin-foil targets with thickness of $<<1 \mu \mathrm{m}$ [14]. They have used a plasma mirror to reduce the 
preformed plasma. Nayuki et al. have reported that the maximum proton energy increased as the product of target thickness and target mass density decreased [15].

In this experiment, we observe the proton signals from thin-foil targets of a polyimide and of copper. We change the thickness of the polyimide target $(7.5 \mu \mathrm{m}$, $12.5 \mu \mathrm{m}$, and $50 \mu \mathrm{m})$ in order to seek the appropriate condition for high-energy proton generation. High-energy protons with the maximum energy of $\sim 2.3 \mathrm{MeV}$ from a $7.5 \mu \mathrm{m}$ thick polyimide target and $\sim 1.2 \mathrm{MeV}$ from a $3 \mu \mathrm{m}$ thick copper target are observed. Here, we employ single-shot time-of-flight (TOF) ion spectrometer and interferometer, which allow us to determine repeatability of the proton acceleration [16, 17].

\section{EXPERIMENTAL SETUP}

We use a Ti:sapphire laser system at The Central Research Institute of Electric Power Industry [18, 19]. In this laser system, the central wavelength is $800 \mathrm{~nm}$ and the pulse duration is 50 fs [full width at half maximum (FWHM)]. The contrast ratio of prepulse at $\sim 5 \mathrm{~ns}$ before the main pulse is measured to be $\sim 10^{-5}$ by a photodiode detector. Figure 1 shows the schematic view of the experimental setup. A p-polarized laser beam is transported into a target chamber and is divided into pump and probe beams by a $5 \mu \mathrm{m}$-thick optical-grade nitrocellulose beam splitter. The pump beam is focused by an off-axis parabolic mirror with a focal length of $f=178.5 \mathrm{~mm}$ and an incidence angle of $45^{\circ}$. The spot size of the focused laser beam is $4 \mu \mathrm{m}$ $(\mathrm{FWHM}) \times 5 \mu \mathrm{m}(\mathrm{FWHM})$. It contains the energy of $\sim 61 \%$ within $1 / \mathrm{e}^{2}$ from the profile of the focusing pattern. The estimated peak intensity is up to $\sim 1 \times 10^{19} \mathrm{~W} / \mathrm{cm}^{2}$ with the energy of $300 \mathrm{~mJ}$ at the target surface. The probe beam passes through the optical delay line. A linear translation stage is used to vary the delay between the pump and probe beams. The plasma image is magnified by a factor of $\sim 10$ and detected by the CCD camera. The resolution in the object plane is approximately $10 \mu \mathrm{m}$. The intensity of the probe beam is attenuated by neutral density filters so as to fall within the dynamic range of the camera. A narrow-band interference filter is placed in front of the CCD cameras to reject unwanted emission from the plasma. The band pass width is $10 \mathrm{~nm}$ for central wavelength of 800 $\mathrm{nm}$. The interferogram is obtained from a Fresnel biprism using a hundred femtosecond probe pulse at the time of $\sim 50$ ps before the pump beam [20,21]. The protons are observed with a TOF ion spectrometer $[16$, 17]. The protons produced by the intense laser are measured in the direction normal to the target. The TOF proton measurement gives on-line real time information of the proton energy distribution $[16,17]$.

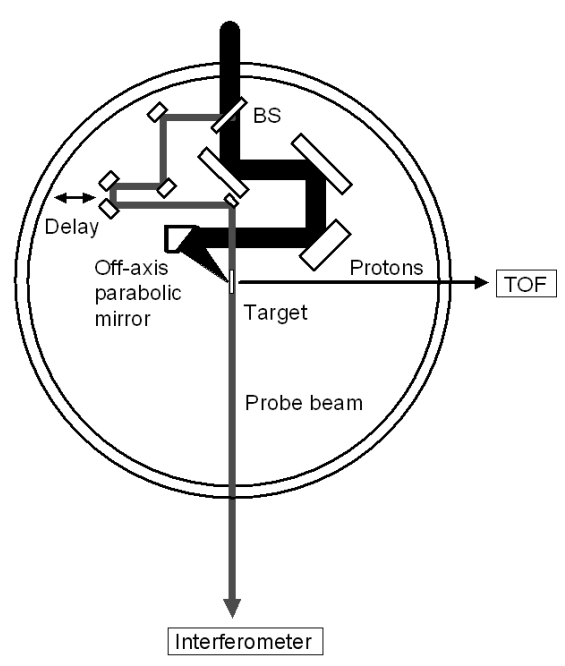

FIG. 1. Schematic diagram of the interferometer: BS stands for the pellicle beam splitter and TOF the timeof-flight ion spectrometer.

\section{EXPERIMENTAL RESULTS}

Figure 2 shows the typical interferogram of the preformed plasma on a $7.5 \mu \mathrm{m}$ thick polyimide target using the probe beam with fundamental wavelength $(800$ $\mathrm{nm})$. They are taken at the time of $\sim 50 \mathrm{ps}$ before the main laser pulse. The arrow shows the direction of the laser pulse propagation. We set the fringe separation to be $\sim 15 \mu \mathrm{m}$ by changing the position of the Fresnel biprism. The original target position is shown by a dashed line. The area of target shadow on the interferogram defines the spatial limit of the fringe visibility. In this case, the intensity of the prepulse at $\sim 5$ ns before the main pulse is $\sim 1 \times 10^{14} \mathrm{~W} / \mathrm{cm}^{2}$. In Fig. 2, the emission at the center of the preformed plasma is observed. It may come from the scattering of the main pulse at the preformed plasma. The probe beam is not able to penetrate into the plasma, whose electron density is up to the critical density, because the refraction in the plasma with steep-density gradient near the original target surface causes significant beam deflection [20]. Fig. 2 shows that the preformed plasma at the front side is generated with the $7.5 \mu \mathrm{m}$ thick polyimide target. No fringe shift is observed with the region $\sim 15 \mu \mathrm{m}$ away from the original surface at the rear side. This shows that the rear side of the target keeps a steep density gradient, which is smaller than the resolution limit of the interferometer of $\sim 15 \mu \mathrm{m}$.

High-energy protons are observed at the rear side of the thin-foil target at the laser intensity of $\sim 1 \times 10^{19}$ $\mathrm{W} / \mathrm{cm}^{2}$. Figure 3 shows the proton energy distributions obtained from the TOF spectra with $7.5 \mu \mathrm{m}$ and 12.5 $\mu \mathrm{m}$ thick polyimide and $3 \mu \mathrm{m}$ thick copper targets. The detection limit of proton energy is about $0.25 \mathrm{MeV}$. The dashed and solid lines show the energy distri- 
butions for $7.5 \mu \mathrm{m}$ and $12.5 \mu \mathrm{m}$ thick polyimide targets in Fig. 3 (a). The maximum proton energy of a $7.5 \mu \mathrm{m}$ thick polyimide target is $\sim 2.3 \mathrm{MeV}$. In the case of 3 $\mu \mathrm{m}$ thick copper target the maximum proton energy is $\sim 1.2 \mathrm{MeV}$ as shown in Fig. 3 (b). The maximum proton energy of a $7.5 \mu \mathrm{m}$ thick polyimide target is higher than that of $3 \mu \mathrm{m}$ thick copper target.

\section{DISCUSSION}

Figure 4 shows the maximum proton energy as a function of the product of material density and thickness. Circles show the $7.5 \mu \mathrm{m}, 12.5 \mu \mathrm{m}$, and $50 \mu \mathrm{m}$ thick polyimide target case. Triangles show the $3 \mu \mathrm{m}$ thick copper target case. Filled symbols are the multi-shot average with error bars corresponding to the standard deviations of shot-to-shot fluctuations. For $50 \mu \mathrm{m}$ polyimide target, the error bar comes from the energy detection limit. Open symbols are the maximum values. We change the target position and plot the maximum proton energy

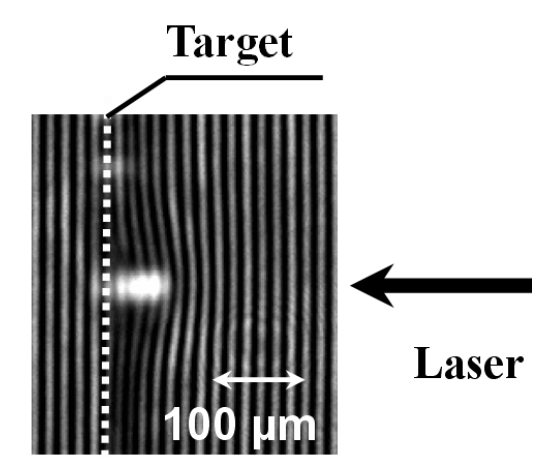

FIG. 2. Interferograms of the preformed plasma for 7.5 $\mu \mathrm{m}$ thick polyimide target with $800 \mathrm{~nm}$ probe beams obtained at $\sim 50$ ps before the main pulse. Dashed lines show the original target position.
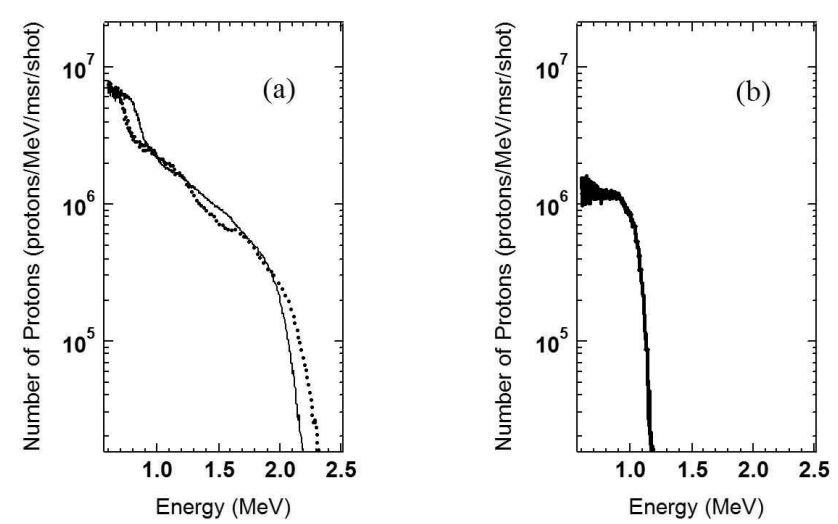

FIG. 3. Energy distribution of proton beam obtained from the TOF signal. (a) The dashed and solid lines show the energy distributions for $7.5 \mu \mathrm{m}$ and $12.5 \mu \mathrm{m}$ thick polyimide targets. (b) The energy distributions for $3 \mu \mathrm{m}$ thick copper target. for each target. Optimum target position is different for each target material. The dashed line shows the detection limit of the maximum proton energy. Please notice those data points below the detection limit mean that no protons are detected. The maximum proton energy decreases according to the value of the product of material density and thickness as shown in Ref. 15. The maximum proton energy of $\sim 2.3 \mathrm{MeV}$ is obtained at the smallest value of the product of target thickness and target mass density in this experimental condition. However, protons are accelerated more stably in the case of large value of the product of material density and thickness; for example, standard deviations of $0.65 \mathrm{MeV}, 0.36 \mathrm{MeV}$, and $0.1 \mathrm{MeV}$ for the value of the product of target thickness and target mass densities of $1.1 \times 10^{-3} \mathrm{~g} / \mathrm{cm}^{2}$, $1.8 \times 10^{-3} \mathrm{~g} / \mathrm{cm}^{2}$, and $2.7 \times 10^{-3} \mathrm{~g} / \mathrm{cm}^{2}$. This is attributed to the smaller influence of ASE and prepulse for large value of the product of material density and thickness.

The laser-driven proton beam has significant features such as delivering an ultra-short and high intensity pulse (high peak current), and having a very small source size and low emittance. The diverging beam has a wide energy spread. Some of these characteristics are advantageous and others are disadvantageous for specific applications. Taking into account such issues, extensive development is going on to realize the applications for industries and medicine using laser driven accelerators. For this purpose we need a high quality, intense and

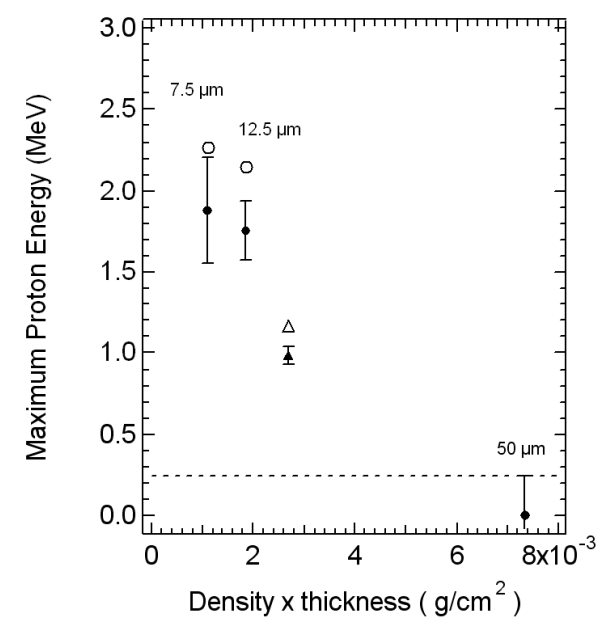

FIG. 4. Maximum proton energy as a function of the product of target density and thickness. Circles show the $7.5 \mu \mathrm{m}, 12.5 \mu \mathrm{m}$, and $50 \mu \mathrm{m}$ thick polyimide target case. Triangles show the $3 \mu \mathrm{m}$ thick copper target case. Filled symbols are the multi-shot average with error bars corresponding to the standard deviations of shot-to-shot fluctuations (For $50 \mu \mathrm{m}$ thickness, the error bar comes from the energy detection limit). Open symbols are the maximum values. The dashed line shows the detection limit of the maximum proton energy. Please notice those data points below the detection limit mean that no protons are detected. 
high-energy proton beam. One of the examples of such a proton beam is reported in Ref. 22. This point proton source with the maximum energy of $4 \mathrm{MeV}$ is applicable to proton radiography. The conversion efficiency (laser energy into the total proton kinetic energy) is $\sim 3 \%$. It is high enough to make radiography using a single laser shot [22].

We have started the Photo-Medical Valley Project in which we are planning to develop a laser driven treatment machine together with a strong collaboration with medical people [23]. Towards the medical applications, we search the laser and target conditions which might be a unique contribution to the industries or sciences.

\section{SUMMARY}

We observe the proton signals with thin-foil targets of a polyimide and of copper. High-energy protons with the maximum energy of $\sim 2.3 \mathrm{MeV}$ from $7.5 \mu \mathrm{m}$ thick polyimide target are observed at the laser intensity of $\sim 1 \times 10^{19} \mathrm{~W} / \mathrm{cm}^{2}$ under the preformed plasma condition. Such protons deliver small emittance beams for various applications such as a proton shadowgraphy. We plan to develop laser-driven proton sources for science, industry, and medical applications.

\section{ACKNOWLEDGMENT}

We would like to thank Dr. T. Tajima, Dr. T. Kimura, Dr. A. Nagashima, and Dr. S. Kawanishi for their support, suggestions, and useful discussions. This work is partly supported by the Special Coordination Fund (SCF) for Promoting Science and Technology commissioned by the Ministry of Education, Culture, Sports, Science and Technology (MEXT) of Japan. This work is partly supported by auspice of Japanese Ministry of Education, Culture, Sports, Science and Technology (MEXT) project on "Mono-energetic quantum beam science with PW lasers". This work is partly supported by JSPS KAKENHI (No. 18540497).

\section{REFERENCES}

1. M. Borghesi, J. Fuchs, S. V. Bulanov, A. J. Mackinnon, P. K. Patel, and M. Roth, "Fastion generation by highintensity laser irradiation of solid targets and applications," Fusion Sci. Technol. 49, 412-439 (2006).

2. H. Daido, M. Nishiuchi, A. Fukumi, Z. Li, A. Sagisaka, K. Ogura, S. Orimo, M. Kado, Y. Hayashi, M. Mori, A. Nagashima, A. Pirozhkov, S. Bulanov, T. Esirkepov, T. Kimura, T. Tajima, K. Nemoto, Y. Oishi, T. Nayuki, T. Fujii, A. Noda, Y. Iwashita, T. Shirai, and S. Nakamura, "Development of laser-driven ion sources," in Proc. Third International Conference on Superstrong Fields in Plasmas (Varenna, Italy, 2005), Proc. AIP conf. vol. 827, pp. 203-214, 2006.

3. M. Murakami, Y. Hishikawa, S. Miyajima, Y. Okazaki, K. L. Sutherland, M. Abe, S. V. Bulanov, H. Daido, T. Zh. Esirkepov, J. Koga, M. Yamagiwa, and T. Tajjima, "Radiotherapy using a laser proton accelerator," in Laserdriven relativistic plasmas applied for science, industry, and medicine, edited by S. V. Bulanov and H. Daido, (Kyoto, Japan, 2007) Proc. AIP conf. (American Institute of Physics) vol. 1024, pp. 275-300, 2008.

4. M. Borghesi, A. J. Mackinnon, D. H. Campbell, D. G. Hicks, S. Kar, P. K. Patel, D. Price, L. Romagnani, A. Schiavi, and O. Willi, "Multi-MeV proton sources investigations in ultraintense laser-foil interactions," Phys. Rev. Lett. 92, 055003 (2004).

5. T. E. Cowan, J. Fuchs, H. Ruhl, A. Kemp, P. Audebert, M. Roth, R. Stephens, I. Barton, A. Blazevic, E. Brambrink, J. Cobble, J. Fernández, J.-C. Gauthier, M. Geissel, M. Hegelich, J. Kaae, S. Karsch, G. P. Le Sage, S. Letzring, M. Manclossi, S. Meyroneinc, A. Newkirk, H. Pépin, and N. Renard-LeGalloudec, "Ultralow emittance, multi$\mathrm{MeV}$ proton beams from a laser virtual-cathode plasma accelerator,” Phys. Rev. Lett. 92, 204801 (2005).

6. M. Nishiuchi, H. Daido, A. Sagisaka, K. Ogura, S. Orimo, K. Kado, A. Yogo, M. Mori, Y. Hayashi, S. Bulanov, A. Fukumi, Z. Li, A. Noda, and S. Nakamura, "Repetitive highly collimated intense proton beam with sub-MeV energy range driven by a compact few terawatt femtosecond laser,” Appl. Phys. B 87, 615-621 (2007).

7. S. Orimo, M. Nishiuchi, H. Daido, A. Yogo, K. Ogura, A. Sagisaka, Z. Li, A. Pirozhkov, M. Mori, H. Kiriyama, S. Kanazawa, S. Kondo, Y. Yamamoto, T. Shimomura, M. Tanoue, Y. Nakai, A. Akutsu, S. Nakamura, T. Shirai, Y. Iwashita, A. Noda, Y. Oishi, K. Nemoto, I. W. Choi, T. J. Yu, J. H. Sung, T. M. Jeong, H. T. Kim, K.-H. Hong, Y.-C. Noh, D.-K. Ko, and J. Lee, "Simultaneous proton and x-ray imaging with femtosecond intense laser driven plasma source,” Jpn. J. Appl. Phys. 46, 5853-5858 (2007).

8. M. Borghesi, A. Schiavi, D. H. Campbell, M. G. Haines, O. Willi, A. J. MacKinnon, L. A. Gizzi, M. Galimberti, R. J. Clarke, and H. Ruhl, "Proton imaging: a diagnostic for inertial confinement fusion/fast ignitor studies," Plasma Phys. Controlled Fusion 43, A267-A276 (2001).

9. F. B. Boody, R. Hopel, and H. Hora, "Laser-driven ion source for reduced-cost implantation of metal ions for strong reduction of dry friction and increased durability, Laser Part. Beams 14, 443-448 (1996).

10. M. I. K. Santala, M. Zepf, F. N. Beg, E. L. Clark, A. E. Dangor, K. Krushelnick, M. Tatarakis, I. Watts, K. W. D. Ledingham, T. McCanny, I. Spencer, A. C. Machacek R. Allott, R. J. Clarke, and P. A. Norreys, "Production of radioactive nuclides by energetic protons generated from intense laser-plasma interactions," Appl. Phys. Lett. 78, 19-21 (2001).

11. K. Nemoto, A. Maksimchuk, S. Banerjee, K. Flippo, G. Mourou, D. Umstadter, and V. Yu. Bychenkov, "Lasertriggered ion acceleration and table top isotope production," Appl. Phys. Lett. 78, 595-597 (2001).

12. M. Kaluza, J. Schreiber, M. I. K. Santala, G. D. Tsakiris, K. Eidmann, J. Meyer-ter-Vehn, and K. J. Witte, "Influence of the laser prepulse on proton accele- 
ration in thin-foil experiments," Phys. Rev. Lett. 93, 045003 (2004).

13. A. J. Mackinnon, Y. Sentoku, P. K. Patel, D. W. Price, S. Hatchett, M. H. Key, C. Andersen, R. Snavely, and R. R. Freeman, "Enhancement of proton acceleration by hot-electron recirculation in thin foils irradiated by ultraintense laser pulses," Phys. Rev. Lett. 88, 215006 (2002).

14. D. Neely, P. Foster, A. Robinson, F. Lindau, O. Lundh, A. Persson, C.-G. Wahlstrom, and P. McKenna, "Enhanced proton beams from ultrathin targets driven by high contrast laser pulses,” Appl. Phys. Lett. 89, 021502 (2006).

15. T. Nayuki, Y. Oishi, T. Fujii, K. Takano, X. Wang, A. A. Andreev, K. Nemoto, and K. Ueda, "Influence of target properties and laser fluence on energetic protons accelerated by a laser-produced plasma,” J. Appl. Phys. 100, 043111 (2006).

16. S. Nakamura, Y. Iwashita, A. Noda, T. Shirai, H. Tongu, A. Fukumi, M. Kado, A. Yogo, M. Mori, S. Orimo, K. Ogura, A. Sagisaka, M. Nishiuchi, Y. Hayashi, Z. Li, H. Daido, and Y. Wada, "Real-time optimization of proton production by intense short-pulse laser with time-of-flight measurement,” Jpn. J. Appl. Phys. 45, L913-L916 (2006).

17. A. Yogo, H. Daido, A. Fukumi, Z. Li, K. Ogura, A. Sagisaka, A. S. Pirozhkov, S. Nakamura, Y. Iwashita, T. Shirai, A. Noda, Y. Oishi, T. Nayuki, T. Fujii, K. Nemoto, I. W. Choi, J. H. Sung, D.-K. Ko, J. Lee, M. Kaneda, and A. Itoh, "Laser prepulse dependency of protonenergy distributions in ultraintense laser-foil interactions with an online time-of-flight technique," Phys. Plasmas 14, 043104 (2007).

18. T. Fujii, Y. Oishi, T. Nayuki, Y. Takizawa, K. Nemoto, T. Kayoiji, K. Horioka, Y. Okano, Y. Hironaka, K. G. Nakamura, and K. Kondo, "MeV-order proton and carbon ion acceleration by irradiation of 60 fs TW laser pulses on thin copper tape," Appl. Phys. Lett. 83, 1524-1526 (2003).

19. Y. Oishi, T. Nayuki, T. Fujii, Y. Takizawa, X. Wang, T. Yamazaki, K. Nemoto, T. Kayoiji, T. Sekiya, K. Horioka, Y. Okano, Y. Hironaka, K. G. Nakamura, K. Kondo, and A. A. Andreev, "Dependence on laser intensity and pulse duration in proton acceleration by irradiation of ultrashort laser pulses on a $\mathrm{Cu}$ foil target," Phys. Plasmas 12, 073102 (2005).

20. A. Sagisaka, H. Daido, K. Ogura, S. Orimo, Y. Hayashi, M. Nishiuchi, M. Mori, K. Matsukado, A. Fukumi, Z. Li, S. Nakamura, K. Takagaki, H. Hazama, M. Suzuki, T. Utsumi, S. V. Bulanov, and T. Esirkepov, "Characterization of preformed plasmas with an interferometer for ultrashort high-intensity laser-plasma interactoins," Appl. Phys. B 78, 919-922 (2004).

21. A. Sagisaka, A. S. Pirozhkov, H. Daido, A. Fukumi, Z. Li, K. Ogura, A. Yogo, Y. Oishi, T. Nayuki, T. Fujii, K. Nemoto, S. Orimo, M. Nishiuchi, Y. Hayashi, M. Mori, M. Kado, S. Nakamura, A. Noda, I. W. Choi, J. H. Sung, D.-K. Ko, and J. Lee, "Development of a two-color interferometer for observing wide range electron density profiles with a femtosecond time resolution,” Appl. Phys. B 84, 415-419 (2006).

22. M. Nishiuchi, H. Daido, A. Yogo, S. Orimo, K. Ogura, J. Ma, A. Sagisaka, M. Mori, A. S. Pirozhkov, H. Kiriyama, S. V. Bulanov, T. Zh. Esirkepov, I. W. Choi, C. M. Kim, T. M. Jeong, T. J. Yu, J. H. Sung, S. K. Lee, N. Hafz, K. H. Pae, Y. -C. Noh, D. -K. Ko, J. Lee, Y. Oishi, K. Nemoto, H. Nagatomo, K. Nagai, and H. Azuma, "Efficient production of a collimated $\mathrm{MeV}$ proton beam from a polyimide target driven by an intense femtosecond laser pulse," Phys. Plasmas 15, 053104 (2008).

23. http://wwwapr.kansai.jaea.go.jp/pmrc_en/ 